\title{
Detection of the quorum sensing signal molecule N-Dodecanoyl-DL-homoserine lactone below 1 nanomolarconcentrations using surface enhanced Raman spectroscopy
}

\author{
Claussen, Anetta; Abdali, Salim; Berg, Rolf W.; Givskov, Michael; Sams, Thomas
}

Published in:

Current Physical Chemistry

Link to article, DOI:

$10.2174 / 1877946811303020010$

Publication date:

2013

Document Version

Early version, also known as pre-print

Link back to DTU Orbit

Citation (APA):

Claussen, A., Abdali, S., Berg, R. W., Givskov, M., \& Sams, T. (2013). Detection of the quorum sensing signal molecule N-Dodecanoyl-DL-homoserine lactone below 1 nanomolarconcentrations using surface enhanced Raman spectroscopy. Current Physical Chemistry, 3(2), 199--214.

https://doi.org/10.2174/1877946811303020010

\section{General rights}

Copyright and moral rights for the publications made accessible in the public portal are retained by the authors and/or other copyright owners and it is a condition of accessing publications that users recognise and abide by the legal requirements associated with these rights.

- Users may download and print one copy of any publication from the public portal for the purpose of private study or research.

- You may not further distribute the material or use it for any profit-making activity or commercial gain

- You may freely distribute the URL identifying the publication in the public portal 


\title{
Detection of the quorum sensing signal molecule $N$-Dodecanoyl-DL-homoserine lactone below 1 nanomolar concentrations using surface enhanced Raman spectroscopy
}

\author{
Anetta Claussen*1, Salim Abdali ${ }^{2}$, Rolf W. Berg ${ }^{3}$, Michael Givskov ${ }^{4}$, and Thomas Sams ${ }^{1}$ \\ ${ }^{1}$ Department of Electrical Engineering, Technical University of Denmark, DK-2800 Lyngby \\ ${ }^{2}$ Biophysical Chemistry Group, Department of Chemistry, H.C. Ørsted Institute, University of \\ Copenhagen, DK- 2100 Copenhagen, Denmark \\ ${ }^{3}$ Department of Chemistry, Energy and Materials, Technical University of Denmark, DK-2800 \\ Lyngby \\ ${ }^{4}$ Department of International Health, Immunology and Microbiology, Panum Instituttet, DK-2200 \\ Copenhagen
}

Keywords: Acyl-Homoserine Lactone, $N$-Dodecanoyl-DL-homoserine lactone, signal molecules, autoinducer, Surface-enhanced Raman spectroscopy, Quorum sensing.

\begin{abstract}
To the best of our knowledge we here for the first time demonstrate surface enhanced Raman spectroscopy (SERS) to detect a quorum sensing (QS) signal molecule below $1 \mathrm{nM}$ concentration in both ultrapure water and under physiological conditions. Based on our results, SERS shows promise as a highly suitable tool for in situ measurements of low Acyl-Homoserine Lactone (AHL) concentrations in biofilms containing QS bacteria. Signal molecules communicate information about their environment and coordinate certain physiological activities in QS systems that exist in many bacteria. SERS enables detection of different AHLs at low concentrations due to structural differences observed in the corresponding SERS spectra. Ag colloidal nanoparticles, produced by the hydroxylamine reducing method, were used for the SERS measurements. SERS spectra of C12-HSL suspended in ultrapure water and in supplemented minimal medium were collected for 5 concentrations ranging from $2 \mu \mathrm{M}$ to $0.2 \mathrm{nM}$, and a comparison between the spectra from these two media is also presented. We have been able to detect biologically relevant concentrations of AHL molecules ranging from $1 \mathrm{nM}$ to $1 \mu \mathrm{M}$ using SERS.
\end{abstract}

\section{Introduction}

Quorum sensing (QS) is an intercellular communication system by which some bacterial cells are capable of indirectly monitoring their own population density through exchange of signal molecules [1]. The expression of virulence factors is kept low until the population density (signal molecule concentration) reaches a threshold value, after which the host system is surprised by a stealth attack [1]. There are several different classes of signal molecules, which include Acyl-Homoserine Lactones (AHLs), autoinducing oligopeptides (AIPs), cyclic dipeptides, such as 2,5-diketopiperazines (DKPs), cholera autoinducer-1 (CAI-1), furanosyl diesters (also called autoinducer-2, AI2), $\gamma$-butyrolactones (GBLs), Pseudomonas quinolone sig- nals (PQSs), and diffusible signaling factors (DSFs) [2]. Furthermore, several hundred structural variants of the basic AHL molecule have been discovered, synthesized, and characterized [2]. In our study here we have looked at the signal molecule known as $N$-Dodecanoyl-DL-homoserine lactone or C12-HSL, where HSL corresponds to homoserine lactone, that originates from the class of AHLs. AHLs are composed of a conserved homoserine lactone and an fatty acid side chain which can vary in length (4-18 carbon atoms), level of saturation, and side-chain (oxo- or hydroxyl-) substitutions [2]. The distribution of the AHLs inside the bacterial cell is thought to depend on two different mechanisms with the short-chain AHLs freely diffusing in/out of the cells and the long-chain AHLs using an active-efflux mechanism to transport themselves in/out 
of the cells [2]. Outside the cell it has been proposed that the long-chain AHLs, like C12-HSL, should not easily be able to move between cells in a diffusion-mediated manner owing to their poorer solubility relative to the shortchain AHLs [2].

The vibrational spectroscopic techniques of infrared (IR) absorption and Raman scattering spectroscopy are both noninvasive methods that yield molecular fingerprint information and have been shown to be a fast and reliable methods to detect and identify/classify bacterial cells [3] 4, 5]. A comparison of the application of the two spectroscopies can be found in Harz et al. and Kirshner et al. [4. 5]. Kirshner et al. found that there is a consistency between the two methods, both spectroscopic techniques are able to be used to discriminate accurately at the strain level, and in addition both techniques showed themselves to be superior to conventional phenotypic methods. Raman spectroscopy has furthermore been used to study the metabolic states of bacteria [6], and the cultivation time dependence of bacterial cellular surface biopolymers [7]. In the work by Bak et al. the infrared spectra of the AHL $\mathrm{N}$-butanoyl-L-homoserine lactone (C4-HSL) in the crystalline form and dissolved in $\mathrm{CCl}_{4}$ have been measured and the IR and the Raman spectra have been calculated using Gaussian03 with the B3LYP hybrid exchange correlation functional (Kohn Sharm, density functional theory) and the cc-pVTZ basis set for the solid state cluster model (a trimer mode)1 [8].

In general, symmetric vibrations give the largest scattering cross section in Raman spectroscopy, while the asymmetric vibrations are the most intense in IR spectroscopy. This arises from what are known as the selection rules: bands which are strongly absorbing in the IR are weak in Raman and vice versa. For centrally symmetric molecules, there is even a mutual exclusion principle. The two techniques are often complementary, and used together, give a better view of the vibrational structure of a molecule. In the infrared, modes/bands which involve large changes in the electric dipole moment vector while in the Raman, modes/bands which involve large changes in the electric dipole - electric dipole polarizability tensor are intense.

A major limitation of Raman spectroscopy is the weakness of the Raman effect which results in very low signals, often below the limit of detection for dilute biological samples. Furthermore it suffers from a strong fluorescence background when lasers are used in the visible region which means that fluorescence from impurities or from the sample itself can mask the Raman spectrum. One can also use lasers at 632.8, 785, 833 and $1064 \mathrm{~nm}$ to alleviate problems due to fluorescence, but since the Raman signal is proportional to $\lambda^{-4}$ the intensity is reduced. IR is much more sensitive than Raman, but its use in biological systems is very limited due to the strong absorbance of water in the fingerprint region, $500 \mathrm{~cm}^{-1}$ to $1800 \mathrm{~cm}^{-1}$. Since we want to measure the spectra of AHLs in aqueous solutions, Raman spectroscopy is more suited for our studies. But since we also want to study very small concentrations, in the range between $10 \mu \mathrm{M}$ and $1 \mathrm{nM}$, an enhancement of the conventional Raman scattering signal is needed. Such enhancements are known to be achieved by e.g. Surface Enhanced Raman Spectroscopy (SERS) [9, 10, 11], Resonance Raman (RR) [12], and Surface Enhanced Resonance Raman Spectroscopy (SERRS) [13]. Resonance Raman occurs when the frequency of the laser beam is close to the frequency of an electronic transition of a chromophore within the system under study. The enhancement is selective for the parts of the molecule involving the chromophore which adsorbs. Often fluorescence is a problem. In SERS the enhancement is a result of the plasmon resonance of the nanoparticle and even single molecules can be detected [14]. In SERS the native fluorescence associated with biological material seen in conventional Raman is suppressed. SERRS uses both the chromophore of RR and the plasmon resonance of SERS to achieve high enhancements, also here single molecules have been detected [13]. Like Raman, SERS has been applied with even greater success to bacteria samples in order to detect, identify and classify bacteria [15, 16, 17, 18].

Pearman et al. have investigated seven different types of signal molecules (differing by acyl chain lengths and varying from 4 to 12 carbons) using SERS with silver colloids, but not in the relevant concentration range, and not under physiological conditions [19]. It was found that all spectra were similar, however, the species with acyl chain length of 4 and 6, i.e. C4-HSL and C6-HSL, differed significantly from the others. For the signal molecule C12HSL the authors were able to detect concentrations between $10 \mu \mathrm{M}$ and $100 \mu \mathrm{M}$, which however is too concentrated to be biologically relevant for our QS system. Our requirement is to be able to detect AHLs at concentrations at least in the nanomolar range.

The C12-HSL molecule has 12 torsion angles that have

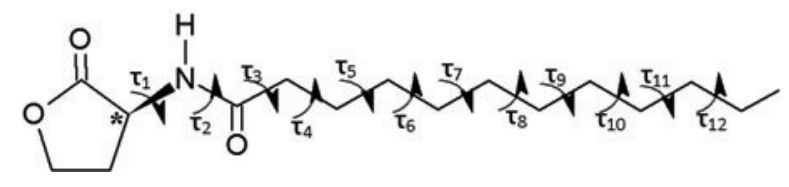

Figure 1: $N$-Dodecanoyl-DL-homoserine lactone, C12HSL, and its 12 torsion angles.

different values in the different conformations that can 


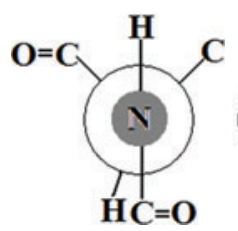

(a)

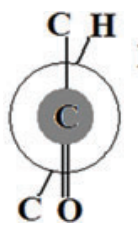

(b)

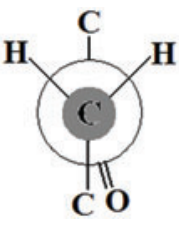

(c)

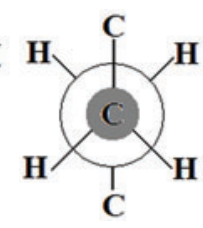

(d)
Figure 2: The Newman projections for the first four torsion angles: $\tau_{1}, \tau_{2}, \tau_{3}$ and $\tau_{4}$.

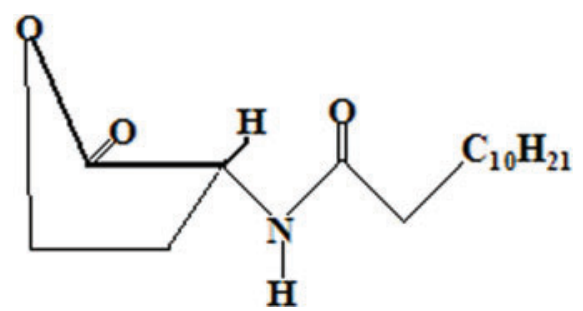

(a)

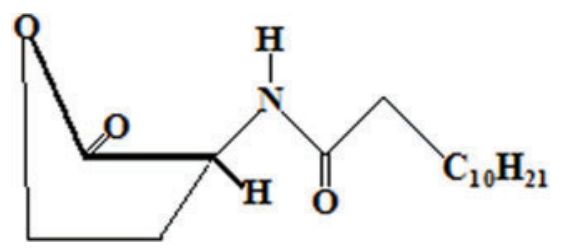

(b)

Figure 3: The ring puckerings, the endo (a) and exo (b) forms.

change in order to place itself in different possible stable conformations, see Figure 1 The Newman projections for the first four torsion angles can be seen in Figure 2 Furthermore, since the molecule has a ketone group there is the possibility of keto and enol tautomerization in addition to the ring puckerings (endo and exo forms). Finally because of the asymmetric carbon (labeled with * in Figure 1) the molecule can be present in L or D states (configurations). Thus there are many structural possibilities. For some of the related AHLs some preferred states have been found. In the work of Bak et al. (using quantum calculations) the presence of two stable conformations, with respect to rotation around the $\mathrm{NC}$ bond linking the butyryl and the lactone moieties, of C4-HSL was revealed, corresponding to endo- and exo-conformations [8], see Figure 3. When including a contribution from a solvent medium by means of the polarized continuum model the preferred state was endo. In another work, Kim et al. investigated C6-HSL comparing quantum calculations with IR-spectroscopies, and found the preferred state also to be endo over exo [20]. In a study by Soulére $e t$ al. the focus was on the acyl chain. They investigated C14-HSL using molecular dynamics and found that the binding mode involved a curved conformation, for more information one is referred to [21].

It has been found that signal molecules can be changed by changes in the environment such as $\mathrm{pH}$, or other species being present such as nitrates, oxidating agents, and degrading enzymes [22]. Alkaline $\mathrm{pH}$ catalyses the AHL hydrolysis, which leads to the lactone ring opening [23]. AHLs with longer acyl chain, such as C12-HSL used in this study, tend to be more resistant to hydrolysis than their shorter chain counterparts [23]. The AHL C6-HSL was shown to convert to several oxo forms when incubated in the presence of nitrates [22]. In the same way, oxidation of AHLs with photo generated $\mathrm{HO}$ was shown to lead to the formation of the corresponding oxo analogues [2]. The reaction of AHLs with oxidants, including hypohalites (salt of a hypohalous acid such as hypoiodite, hypobromite, hypochlorite, or hypofluorite.) and hydroxyl radicals, can result in attenuation of AHLs by oxidation and subsequent inhibition of QS [2]. AHLs without additional functional groups on the acyl chain, such as C12HSL, have been shown to be nonreactive to added hypohalites, while all AHLs were reactive with the hydroxyl radical.

Here we show that it is possible to acquire SERS spectra of C12-HSL from $\mu \mathrm{M}$ down to concentrations as low as $0.2 \mathrm{nM}$. This concentration range is well within the biological concentrations found in bacteria, the threshold typically being in the nanomolar range [2]. These spectra were acquired not only in aqueous solution, but also in a solution mimicking physiological conditions. The spectra are seen not to be quantitative, they do not appear to follow Beers law for their concentrations. This requires further investigation.

\section{Materials and method}

\subsection{Materials}

The $N$-Dodecanoyl-DL-homoserine lactone (C12-HSL) used in this study was purchased from Sigma-Aldrich. The molecular structure, the Newman projections for the first four torsion angles and the ring puckerings for the lactone ring (endo and exo form) can be seen in Figures 1 , 2 and 3. A conventional Raman spectrum measured with a wavelength of $532 \mathrm{~nm}$ is seen in Figure 4 . Supplementary minimal medium, ABtGcasa, was used as the physiological environment. $\mathrm{ABtG}$ casa is made from $\mathrm{ABt}(\mathrm{B}$ medium [24] plus $2.5 \mathrm{mg} / \mathrm{l}$ thiamine and $10 \%$ A10 [24]) supplemented with $0.5 \%$ glucose and $0.5 \%$ Casa amino acids. 
Water used in the experiments was ultrapure water from the Milli-Q Ultrapure Water System. Referring to ethanol it is always ethanol $96 \%$.

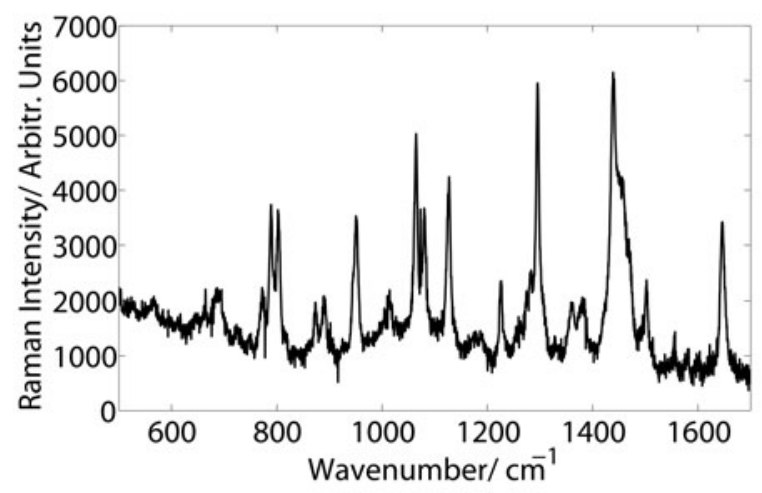

Figure 4: Conventional Raman spectra of $N$-DodecanoylDL-homoserine lactone (C12-HSL) in the solid phase using a $532 \mathrm{~nm}$ wavelength laser, same as used for the SERS measurement.

\subsection{Instrumentation}

Surface-enhanced Raman spectra were recorded with the use of a frequency doubled $\mathrm{Nd}: \mathrm{YVO}_{4}$ laser at an excitation wavelength of $532 \mathrm{~nm}$. Output power for all measurements was $250 \mathrm{~mW}$. The experiments were performed with a dispersive Dilor-XY Raman spectrometer. Raman spectra were obtained using a liquid nitrogen cooled CCD using the Labspec software. The Raman spectrum of cyclohexane $\left(\mathrm{C}_{6} \mathrm{H}_{12}\right)$, which is an alkane widely used to calibrate spectrometers since it exhibits very strong Raman lines at $801 \mathrm{~cm}^{1}$ and $2852 \mathrm{~cm}^{1}$, was used for validation and calibration purposes. The band at $801 \mathrm{~cm}^{1}$ was used for calibration. All spectra were collected with an exposure time of $6.7 \mathrm{sec}$ and averaged over 2 accumulations. No baseline or background corrections were applied to the spectra shown. Gas chromatography target vials were used to contain the samples in the SERS measurements. The target vials were made from glass to ensure minimum interference in the spectra and had a diameter of $9 \mathrm{~mm}$. Ultraviolet visible (UV-VIS) extinction data was collected using a Shimadzu UV-1800 spectrophotometer. A quartz cuvette with a path length of $10 \mathrm{~mm}$ was used.

\subsection{Ag preparation}

The silver colloids used here for the SERS measurements were produced by reduction of $\mathrm{AgNO}_{3}$ by hydroxylamine ions, following the method of Leopold et al. [25]. Under a steady stirring and at room temperature, $90 \mathrm{ml}$ of $3.3 \mathrm{mM}$ $\mathrm{NaOH}$ was mixed with $0.5 \mathrm{ml}$ of $0.3 \mathrm{M}$ hydroxylamine hydrochloride $(\mathrm{HA} \mathrm{HCl})$ and $10 \mathrm{~mL}$ of $0.01 \mathrm{M} \mathrm{AgNO}_{3}$. The reduction took place immediately and the colloids were examined by UV-VIS and checked by Scanning Electron Microscope, SEM. The extinction spectrum of the resulting Ag hydrosol can be seen in Figure 5. The spectral top is found to be $\lambda_{\max }=405.25 \mathrm{~nm}$ and the full width at half maximum (FWHM) is measured to be $68 \mathrm{~nm}$.

For nanorods and nanoparticle aggregates, the plasmon

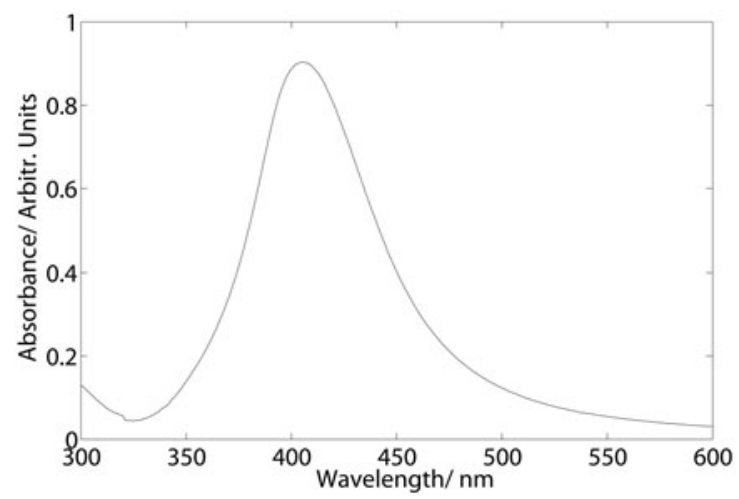

Figure 5: UV-VIS extinction spectrum of Ag colloids produced via hydroxylamine reduction method. A 9 times dilution is used.

absorption splits into two bands corresponding to the oscillation of the free electrons along and perpendicular to the long axis of the rods [26, 27, 28]. Since we only have one peak we therefore assume that the particles have a roughly spherical shape.

The absorption maximum of the measured UV-VIS spectrum of the colloidal solution provides information on the average particle size, whereas its FWHM can be used to estimate particle dispersion [25, 29]. For silver particles with a diameter smaller than $10 \mathrm{~nm}$ dipolar absorption is dominant [29, 30] and one can use the dipole approximation from Mie scattering theory to find a simple relationship between the FWHM and the radii of the particle. This approximation gives a band of Lorentzian shape. For larger radii particles, the absorption band begins to shift to longer wavelengths due to additional magnetic-dipole terms, thus the full expression of Mie's theory applies. The extinction spectra obtained for the silver particles are quite broad with a tail on the higher wavelength side of the peak, see Figure 5. The tail could be due to the dipolar scattering and quadrupole absorption, and it indicates that the size of the particles must be large [31]. Since the absorption maximum is $405.25 \mathrm{~nm}$ the mean diameter 
of the silver colloid particles can be assumed to be approximately $23 \mathrm{~nm}$ according to [25] which uses the same production method as this work. Also from [32] the mean diameter can be assumed to be between 20 and $24 \mathrm{~nm}$. This is validated by SEM.

\subsection{Sample preparation}

$N$-Dodecanoyl-DL-homoserine lactone (C12-HSL) is only slightly soluble in water mainly due to the hydrophobic nature of the acyl chains. Thus, we decided to dissolve the solid C12-HSL in an organic solvent and thereafter dilute it with ultrapure water. The solvent chosen had to be without significant structure in the fingerprint region of the Raman spectrum $\left(500-1800 \mathrm{~cm}^{-1}\right)$. We considered dimethyl sulfoxide (DMSO) used by Pearman et al.[19] and ethanol. Ethanol was chosen because of its weaker spectrum as compared to the DMSO spectrum. A stock solution of $10 \mathrm{mM}$ was prepared by dissolving $1 \mathrm{mg}$ of C12-HSL in $353 \mu \mathrm{l}$ of $96 \%$ ethanol. A part of this stock solution was added to an eppendorf tube containing ultrapure water or ABtGcasa medium and manually shaken. The final C12-HSL concentrations ranged from $2 \times 10^{-10}$ $\mathrm{M}$ to $2 \times 10^{-6} \mathrm{M}$ both in ultrapure water and in ABtGcasa media.

\subsection{Setup and spectra}

In order to achieve the best condition, which yields highest peak intensity, optimization of the volume ratio of the sample at $2 \mu \mathrm{M}$ concentration to the colloids was carried out, using the same approach as in [33]. The volume ratios tested was $40 \%$ sample to $60 \%$ colloids, $50 \%$ sample to $50 \%$ colloids, $60 \%$ sample to $40 \%$ colloids, $70 \%$ sample to $30 \%$ colloids, and $80 \%$ sample to $20 \%$ colloids for the C12-HSL in water. For C12-HSL in ABtGcasa the volume ratios tested was $30 \%$ sample to $70 \%$ colloids, $40 \%$ sample to $60 \%$ colloids, $50 \%$ sample to $50 \%$ colloids, $60 \%$ sample to $40 \%$ colloids. For C12-HSL in water the spectra with the highest intensity was seen for the $60 \%$ sample to $40 \%$ colloids, and for the C12-HSL in ABtGcasa the $40 \%$ sample to $60 \%$ colloids gave the highest intensity.

The final AHL concentrations were $2 \times 10^{-10} \mathrm{M}, 2 \times 10^{-9}$ M, $2 \times 10^{-8} \mathrm{M}, 2 \times 10^{-7} \mathrm{M}$ and $2 \times 10^{-6} \mathrm{M}$ for samples in water and ABtGcasa medium. Our smallest concentrations is $0.2 \mathrm{nM}$ which is the equivalent of $0.2 \times 10^{-9}$ mol/l or $1.2 \times 10^{14}$ molecules per liter. The recording parameters were kept the same in all these measurements including the sample volume of $200 \mu \mathrm{L}$.

The sample diluted in ABtGcasa medium was controlled at a $\mathrm{pH}$ of 6.3 due to the A-10 buffer in the ABtGcasa solution. The $\mathrm{pH}$ in the sample diluted in water is assumed to be approximately 7 , which is the $\mathrm{pH}$ of the colloid solution [25]. It is known that under alkaline conditions AHL's are susceptible to hydrolysis [2]. AHLs having longer acyl-chain (C12-C14) are significantly less susceptible to hydrolysis of the lactone, the half-life for C12-HSL being between $1(\mathrm{pH}=9.55)$ and 64 hours [ [23], 27 hours for a $\mathrm{pH}$ of 7.2. All sample solutions were measured within hours to limit any possible hydrolysis of the parent AHL.

\section{Quantum lactone ring model}

Quantum chemical calculations were performed with the GAUSSIAN 09 program [34] of a simplified acyl-HSL with only two carbon atoms in the acyl chain, called C2HSL. Three versions of C2-HSL were compared to find the energy minimum: protonation on the amide carbonyl, protonation on the lactone carbonyl and the unprotonated C2-HSL, see Figure 6. The LC-wPBE density functional [35] combined with the 6-31Gdp [36, 37] basis set were used and the energy minimum was found for the unprotonated version, see Table 3. Hence, the B3PW91 den-

\begin{tabular}{ll}
\hline & Table 1: Energies \\
\hline structure & $\Delta \mathrm{E}[\mathrm{kcal} / \mathrm{mol}]$ \\
\hline UP & 0 \\
PL & 17.811859 \\
PA & 51.24348
\end{tabular}

Table 1: Energy of the C2-HSL in the three states: protonation on the amide carbonyl (PA), protonation on the lactone carbonyl (PL) and the unprotonated C2-HSL (UP)

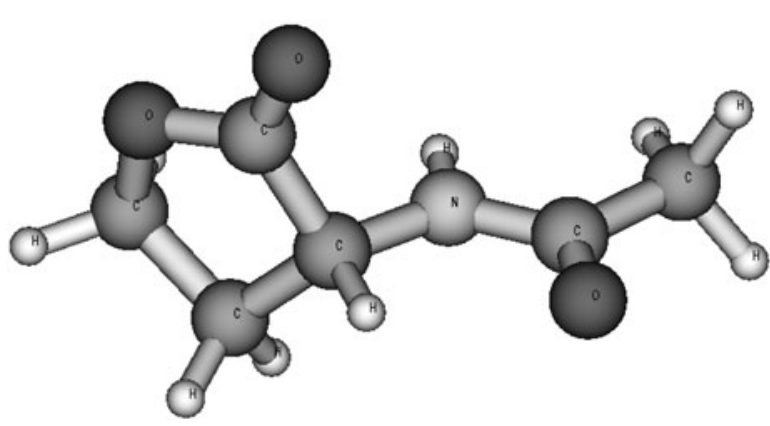

Figure 6: The optimized form of the unprotonated C2HSL. 
sity functional [38] and the 6-31Gdp basis set was used to obtain the Raman spectra of the unprotonated C2-HSL, which can be seen in Figure 7 The theoretical wave num-

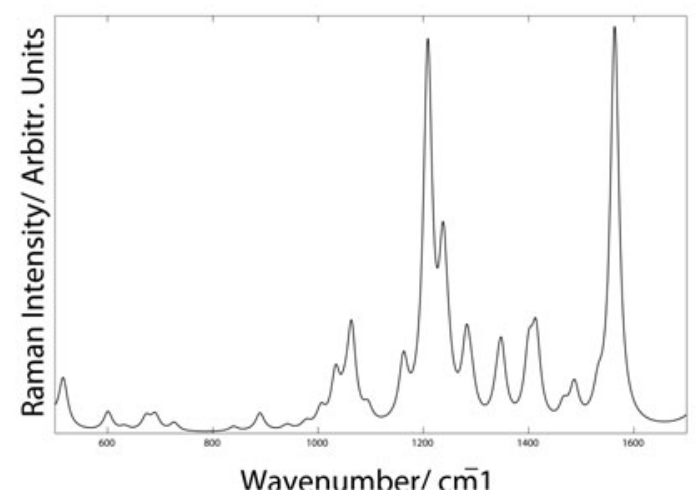

Figure 7: The theoretical Raman spectra of the unprotonated C2-HSL. The wave numbers are scaled by the factor of 0.9602 and a Lorentz lineshape with HWHM at 10 $\mathrm{cm}^{-1}$ is assumed.

bers are scaled by the factor of 0.9602 [45] and a Lorentz lineshape with HWHM $=10 \mathrm{~cm}^{-1}$ is assumed.

\section{Discussion and results}

The measured SERS spectra for C12-HSL with the range of concentrations given in the Materials and Methods section are presented in Figure 8 and 9 . In these Figures the highest concentration is depicted in the top, decreasing in concentration towards the bottom, the concentrations being $20 \mu \mathrm{M}, 2 \mu \mathrm{M}, 200 \mathrm{nM}, 20 \mathrm{nM}, 2 \mathrm{nM}, 0.2 \mathrm{nM}$ and 0 $\mathrm{M}$. The lowest spectrum is the control spectrum, that is, the spectrum of the same sample without C12-HSL. Figure 8 shows the spectra of C12-HSL dissolved in water and Figure 9 shows the spectra of C12-HSL dissolved in ABtGcasa.

The proposed assignment of the spectrum can be seen in Table 2 and shows all peaks observed both for the C12HSL in water and in ABtGcasa and it is indicated at which concentrations the peaks were seen. Furthermore, Table 2 shows the positions at which we found ethanol and ABtGcasa in order to see whether these could interfere with the C12-HSL spectra. The C12-HSL spectra are dominated by contributions from the amide portion of the molecule, that is the majority of the strong spectral bands originates from the chain of C12-HSL. This was also seen in the work of Pearman et al. [19]. The vibrations attributed to the lactone ring are weak, except for the very strong vibration at $1363 \mathrm{~cm}^{-1}$ which is the $\mathrm{CH}_{2}$ wagging vibra-

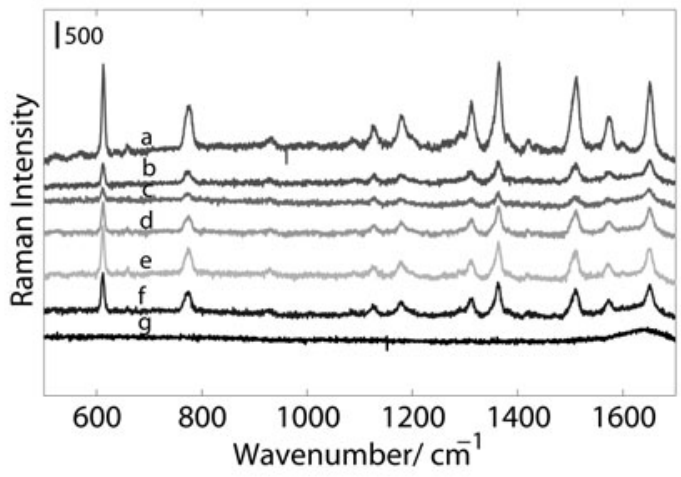

Figure 8: SERS of C12-HSL suspended in ultrapure water for the following concentrations (top down): $20 \mu \mathrm{M}$ (a), 2 $\mu \mathrm{M}$ (b), $200 \mathrm{nM}$ (c), $20 \mathrm{nM}$ (d), $2 \mathrm{nM}$ (e) and $0.2 \mathrm{nM}$ (f). The lowest spectrum (g) is without any C12-HSL, thus only ultrapure water and $\mathrm{Ag}$ colloids are present.

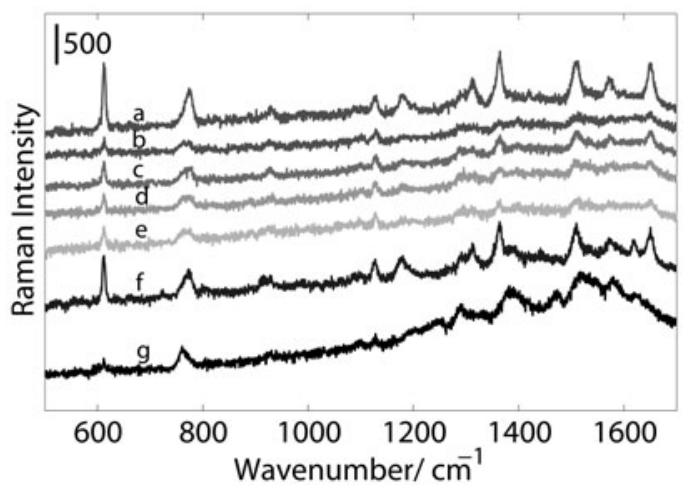

Figure 9: SERS of C12-HSL suspended in ABtGcasa medium for the following concentrations (top down): 20 $\mu \mathrm{M}$ (a), $2 \mu \mathrm{M}$ (b), $200 \mathrm{nM}$ (c), $20 \mathrm{nM}$ (d), $2 \mathrm{nM}$ (e) and 0.2 $\mathrm{nM}(\mathrm{f})$. The lowest spectrum (g) is without any C12-HSL, thus only ABtGcasa medium and Ag colloids are present.

tion of the lactone ring according to the quantum chemical calculation conducted in this study and also according to Jakubczyk et al. [47]. Furthermore, the medium band seen at $1178 \mathrm{~cm}^{-1}$ is attributed to the C-O stretch of the lactone ring. The very strong amide $\mathrm{I}$ band at 1650 $\mathrm{cm}^{-1}$ could also contain contributions from $\mathrm{C}=\mathrm{O}$ in lactone. The amide I, II, III and IV bands at $1650 \mathrm{~cm}^{-1}$, $1573 \mathrm{~cm}^{-1}, 1312 \mathrm{~cm}^{-1}$ and $773 \mathrm{~cm}^{-1}$, respectively are all clearly identifiable for C12-HSL in the water solution while the amide II and III are less pronounced for the $\mathrm{C} 12$ HSL spectra in ABtGcasa for all concentrations except the highest, $20 \mu \mathrm{M}$ where they are very clear. The band for the 
Table 2: Assignment C12-HSL

\begin{tabular}{|c|c|c|c|c|c|c|c|}
\hline $\begin{array}{l}\tilde{v}_{\text {Ethanol }} \\
{\left[\mathrm{cm}^{-1}\right]}\end{array}$ & $\begin{array}{l}\tilde{\mathrm{v}}_{A B \mathrm{~B} t \text { Gcasa }} \\
{\left[\mathrm{cm}^{-1}\right]}\end{array}$ & $\begin{array}{l}\tilde{\mathrm{v}}_{\text {solid }} \\
{\left[\mathrm{cm}^{-1}\right]}\end{array}$ & $\begin{array}{l}\tilde{\mathrm{v}}_{\mathrm{calc}} \\
{\left[\mathrm{cm}^{-1}\right]}\end{array}$ & $\begin{array}{l}\tilde{\mathrm{V}}_{S E R S} \\
{\left[\mathrm{~cm}^{-1}\right]}\end{array}$ & Observed in & Assignment & Ref. \\
\hline & & 523 & 496 & $523_{\mathrm{vw}}$ & $1,2,4,5,6, \mathrm{a}, \mathrm{f}$ & $\mathrm{O}=\mathrm{C}-\mathrm{C}$ (lactone) scissoring, $\mathrm{N}-\mathrm{H}$ def & calc \\
\hline & & 566 & 578 & $573_{v w}$ & $1.5,6, \mathrm{f}$ & $\mathrm{C}-\mathrm{C}=\mathrm{O}$ scissoring $/ \mathrm{C}-\mathrm{C}$, chain & calc/[19, 47] \\
\hline & 612 & & & $612_{\mathrm{vs}}$ & $1,2,3,4,5,5,6$ a,b,c,d,e,f & $\mathrm{O}=\mathrm{C}-\mathrm{N}$ bending & [19,47] \\
\hline & & & 647 & $6399_{\mathrm{vw}}$ & $1,2, \mathrm{a}, \mathrm{e}, \mathrm{f}$ & C-C-C symmetric stretch & calc \\
\hline & & 664 & 662 & $660_{w}$ & $1,2,3,4,5,5$, a, a, f & asymmetric stretch ring & calc \\
\hline & & $747-687$ & & & & & \\
\hline & 760 & $802-772$ & & 773 s & $1,2,3,4,5,6,6, \mathrm{a}, \mathrm{b}, \mathrm{c}, \mathrm{d}, \mathrm{e}, \mathrm{f}$ & amide IV, & [19, 47 \\
\hline & & 888-874 & 856 & & & $\begin{array}{l}\mathrm{C}=\mathrm{O} \text { deformation, } \mathrm{N}-\mathrm{H} \text { bend } \\
\text { ring stretch symmetric }\end{array}$ & calc \\
\hline \multirow[t]{3}{*}{$880_{\mathrm{vs}}$} & 928 & & & $929 \mathrm{w}$ & $1,2,3,4,5,5,6, \mathrm{a}, \mathrm{b}, \mathrm{c}, \mathrm{de}, \mathrm{f}, \mathrm{f}$ & $\mathrm{C}-\mathrm{O}-\mathrm{C}$ assymetric stretching (cyclic) & [19] \\
\hline & & 950 & 967 & & & $\mathrm{CH}_{2}$ wagging & calc \\
\hline & & 1014 & 992 & & & $\mathrm{CH}$ def & calc \\
\hline \multirow{5}{*}{$\begin{array}{l}1052_{\mathrm{m}} \\
1094_{\mathrm{m}}\end{array}$} & & 1065 & 1021 & $1010_{\mathrm{vw}}$ & 1,6 & C-C-C stretch, lactone & [19, 47] \\
\hline & 1098 & 1080 & 1050 & $1093_{w}$ & $1,2,4,5,5, \mathrm{a}, \mathrm{ab}, \mathrm{b}, \mathrm{d}, \mathrm{e}, \mathrm{f}, \mathrm{g}$ & $\mathrm{CH}_{2}$ rocking, lactone & [19, 47] \\
\hline & 1128 & 1127 & 1117 & $1128 \mathrm{~m}$ & $1,2,3,4,5,6,6, \mathrm{ab}, \mathrm{b}, \mathrm{d}, \mathrm{e}, \mathrm{f}$ & $\mathrm{C}-\mathrm{H}$ def./C-C-C & calc \\
\hline & 1197 & 1178 & 1161 & $1178_{\mathrm{m}}$ & $1,2,3,4,5,6,6, \mathrm{ab}, \mathrm{b}, \mathrm{d}, \mathrm{e}, \mathrm{f}$ & C-O stretch(lactone) & calc,[47] \\
\hline & 1246 & 1191 & 1188 & $1201_{\mathrm{vw}}$ & $1,2,5, \mathrm{a}, \mathrm{c} \mathrm{c}$ & $\mathrm{CH}_{2}$ rocking & [46], calc \\
\hline \multirow[t]{5}{*}{$1277_{\mathrm{w}}$} & 1293 & & & $1291_{\mathrm{vw}}$ & $1,5,6, \mathrm{a}, \mathrm{c}, \mathrm{d}, \mathrm{d}, \mathrm{f}, \mathrm{f}$ & $\mathrm{CH}_{2}$ wagging+bending, lactone & {$[47$} \\
\hline & 1313 & 1296 & 1294 & $1312_{s}$ & $1,2,3,4,5,6,6, \mathrm{a}, \mathrm{b}, \mathrm{c}, \mathrm{d}, \mathrm{e}, \mathrm{f}$ & amide III, C-N def. & [19] \\
\hline & 1333 & 1359 & 1356 & $1363_{\text {vs }}$ & 1,2,3,4,5,5,6,a,b,c,d,e,f & $\mathrm{CH}_{2}$ wagging, lactone & [47], calc \\
\hline & 1383 & & & $1382_{\mathrm{vw}}$ & $1,4,5, \mathrm{~b}, \mathrm{c}, \mathrm{d}, \mathrm{e}, \mathrm{f}$ & $\mathrm{CH}_{2}$ wagging, lactone & [47],calc \\
\hline & & & & $1419_{\mathrm{vw}}$ & 1,2,4,5,6,a & C-N stretch & [47] \\
\hline \multirow[t]{8}{*}{$1455_{s}$} & & 1380 & 1411 & $1439_{\mathrm{w}}$ & 1 & $\mathrm{CH}_{2}$ scissoring, lactone & [19, 47] \\
\hline & 1474 & 1439 & 1427 & $1469_{\mathrm{vw}}$ & $1,2,5,6, \mathrm{f}$ & $\mathrm{CH}_{2} / \mathrm{CH}_{3}$ deformation, chain & {$[19] /$ calc } \\
\hline & 1529 & & & $1511_{\text {vs }}$ & $1,2,3,4,5,5,6, \mathrm{a}, \mathrm{b}, \mathrm{c}, \mathrm{d}, \mathrm{e}, \mathrm{f}$ & NH bending & [19, 47] \\
\hline & 1580 & 1503 & 1501 & $1573_{s}$ & $1,2,3,4,5,5,6, \mathrm{a}, \mathrm{b}, \mathrm{c}, \mathrm{d}, \mathrm{e}, \mathrm{f}$ & $\begin{array}{l}\text { amide II, NH def. } \\
\text { (trans co-planar) }\end{array}$ & [19], calc \\
\hline & & & & $1601_{\mathrm{w}}$ & $1,2,4,5,6,6, \mathrm{a}$ & $\mathrm{NH}$ deformation & [19] \\
\hline & 1622 & & & $1620_{s}$ & $\mathrm{f}$ & ABtGcasa & \\
\hline & & 1646 & 1690 & $1650_{v s}$ & $1,2,3,4,5,5,6, \mathrm{ab}, \mathrm{b}, \mathrm{d}, \mathrm{de}, \mathrm{f}$ & amide $\mathrm{I}, \mathrm{C}=\mathrm{O}$ ketone stretch & [19, 47], calc \\
\hline & & 1779 & 1784 & & & $\mathrm{C}=\mathrm{O}$ lactone stretch & [47], calc \\
\hline
\end{tabular}

Table 3: Intensity codes: $\mathrm{v}=$ very, $\mathrm{w}=\mathrm{weak}, \mathrm{m}=$ medium, $\mathrm{s}=$ strong.

$20 \mu \mathrm{M}=1, \mathrm{a}, 2 \mu \mathrm{M}=2, \mathrm{~b}, 200 \mathrm{nM}=3, \mathrm{c}, 20 \mathrm{nM}=4, \mathrm{~d}, 2 \mathrm{nM}=5, \mathrm{e}, 0.2 \mathrm{nM}=6, \mathrm{f}$

solid, refers to the Raman spectrum of the solid crystal C12-HSL, calc refers to the calculated Raman spectrum of the C2-HSL using GAUSSIAN 09 with B3PW91/6-31Gpd, and SERS refers to the SERS spectra of the C12-HSL both in water and ABtGcasa. 
$\mathrm{O}=\mathrm{C}-\mathrm{N}$ bending vibration at $612 \mathrm{~cm}^{-1}$ is consistently the strongest band observed in the SERS spectra of C12-HSL both for the water solution and the ABtGcasa-solution.

The control spectrum for the water solution is featureless, see Figure $8 \mathrm{~g}$ ). Clearly a reduction of the water background is seen, which is due to the adsorption by the silver nanoparticles. We chose to obtain spectra of C12-HSL in ABtGcasa solution, because this is a typical environment in which to grow bacteria. In this way we tested whether the solution interfered with the spectrum. A number of peaks were seen in the ABtGcasa but these peaks do not seem to dominate in the combined spectra, see Figure $9 \mathrm{~g}$ ). However, many of the ABtGcasa-baseline peaks are positioned in similar areas as the C12-HSL peaks; this may result in the peaks in the C12-HSL spectra dissolved in ABtGcasa having an extra contribution from the ABtGcasa media. When comparing with the water dissolved C12-HSL spectra it can indeed be seen that the amide IV band at $773 \mathrm{~cm}^{-1}$ is broader since this band coincides with a band in the ABtGcasa spectra at an identical position. Because of the enhanced fluorescence caused by ABtGcasa to the right part of the spectra, see Figure 9 the peaks in this part are less enhanced. Furthermore, the majority of peaks in the ABtGcasa spectra lie in this right hand-side of the spectra causing the peaks of the C12-HSL to be broader and less identifiable. When inspecting the lowest concentration (Figure 9 $\mathrm{g}$ )) an additional peak can be seen at the wavelength $1620 \mathrm{~cm}^{-1}$. This is proposed to be due to the ABtGcasa contribution which has a peak at $1622 \mathrm{~cm}^{-1}$. Why it is not present for the higher concentrations could be due to the ratio of ABtGcasa to $\mathrm{C} 12$ HSL being much higher for low C12-HSL concentration. There is simply a much higher risk that ABtcasa bands are enhanced. The comparison of the C12-HSL in the two solutions at the lowest concentration can be seen in Figure 10. In this figure the peak at $1620 \mathrm{~cm}^{-1}$ proposed to be from the ABtGcasa solution is clearly seen.

As has been mentioned the strongest band in the C12-HSL spectra is the $\mathrm{O}=\mathrm{C}-\mathrm{N}$ band at $612 \mathrm{~cm}^{-1}$ both for the water and the ABtGcasa solution. However, the strongest band amongst the amide bands differs between the two solutions. For the water solution the strongest amide band is the amide I band while the strongest band for the ABtGcasa solution is the amide IV band, closely followed by the amide III band. The weakest band in both solutions is the amide II band. This suggest that the $\mathrm{O}=\mathrm{C}-\mathrm{N}$ band experiences a greater SERS enhancement and thus would also suggest an orientation of the molecule where the $\mathrm{O}=\mathrm{C}-\mathrm{N}$ is both closer to and nearly normal to the metal surface, thus experiencing maximum enhancement. That the second strongest band differs between the two solu-

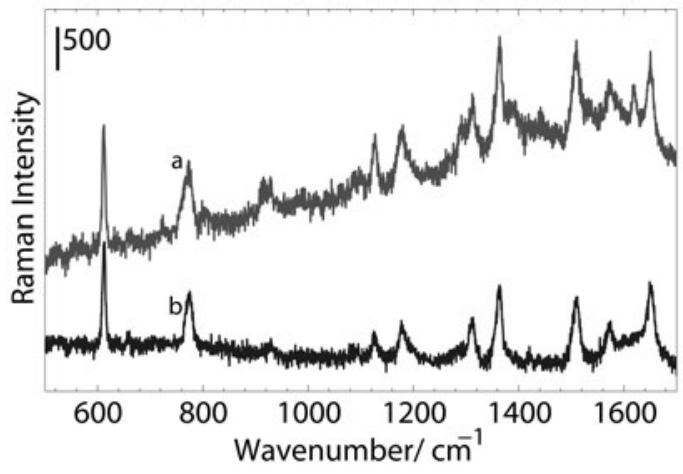

Figure 10: SERS of C12-HSL suspended in ABtGcasa medium (a: red) and for C12-HSL suspended in ultrapure water (b: blue) at $0.2 \mathrm{nM}$. The spectra of the lowest concentrations from Figure 4 (b:blue) and Figure 5 (a:red).

tions could mean that the orientation of the molecule adsorbed to the nanoparticle has changed.

As mentioned in the section, Setup and spectra the $\mathrm{pH}$ of the $\mathrm{C} 12-\mathrm{HSL}$ in ABtGcasa is assumed to be approximate 7 , but it is not fixed and thus could be higher. At higher pH C12-HSL could undergo hydrolysis, resulting in opening of the lactate ring. In the study of Pearman et al. hydrolysis was investigated and the following new additional peaks observed: $1270 \mathrm{~cm}^{-1}$ attributed to carboxylic CO stretch band and $1432 \mathrm{~cm}^{-1}$ to a combination band, the latter due to carboxylic $\mathrm{CO}$ stretch and $\mathrm{OH}$ deformation [19]. None of these bands are found in the spectra in our study, thus we assume that no hydrolysis is occurring. Since hydrolysis halftime is found to be 27 hours for a $\mathrm{pH}$ of 7.2 and the longer acyl chain of C12-HSL makes it less susceptible to hydrolysis, we would not expect to see any signs of hydrolysis in our experimental conditions.

When inspecting the spectra of the C12-HSL in water in Figure 8 the intensity of the spectra can be seen not to drop with decreasing concentration. The highest intensity is found in the spectrum for the highest concentration, 20 $\mu \mathrm{M}$. From $20 \mu \mathrm{M}$ the intensity of all the peaks decreases with concentration until the concentration of $200 \mathrm{nM}$. At $20 \mathrm{nM}$ the intensity level rises above the intensity levels of $200 \mathrm{nM}$ and $2 \mu \mathrm{M}$. The intensity rising for $2 \mathrm{nM}$ and hereafter decreasing for $0.2 \mathrm{nM}$. This non-linearity can be seen in Figure 11. The peak height at $612 \mathrm{~cm}^{-1}$, corresponding to the $\mathrm{O}=\mathrm{C}-\mathrm{N}$ bending vibration and the peak heights of the amide I-IV bands, are followed and plotted against the different concentrations. All monitored peaks follow the same trend. In the case of C12-HSL suspended in water the intensity of the spectra seems to stop decreasing at around the concentration $200 \mathrm{nM}$. The 


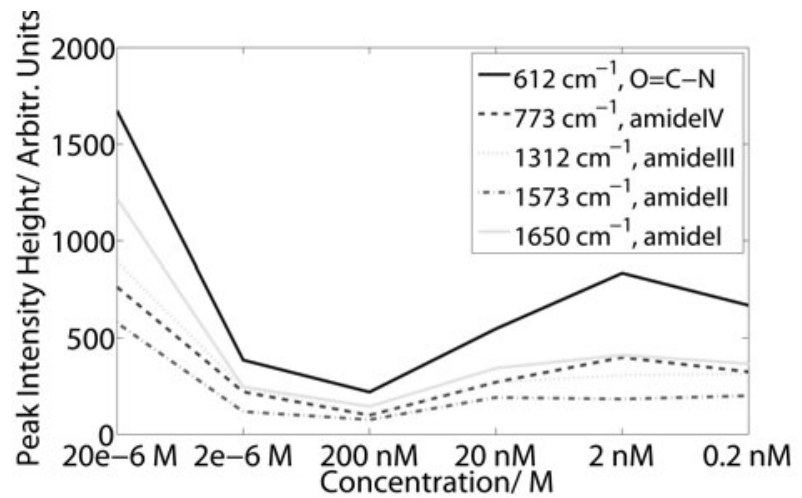

Figure 11: The peak intensity height at $612 \mathrm{~cm}^{-1}$, corresponding to the $\mathrm{O}=\mathrm{C}-\mathrm{N}$ bending vibration, is followed through the different concentrations and plotted against the concentration. Likewise are the amide bands. Here we have the plot for C12-HSL in water solution.

decrease in intensity with falling concentration until 200 $\mathrm{nM}$ is what one would expect because the silver surface area has less molecules adsorbed. However the fall can also be seen to be non-linear, which is seen in literature and thus why a small concentration range is often chosen [40, 42]. After $200 \mathrm{nM}$ the intensity increases until $2 \mathrm{nM}$. The increase in enhancement for the low concentrations could be explained by the existence of hot spots in the cluster structures, caused by thermally and non thermally activated diffusion of molecules into and out of hot areas, or trapping and release of molecules in high-field gradients [39]. It could also be due to the different adsorption geometries that can exist at different concentrations, as seen in [40, 41]. A major problem in SERS is not only the difficulty in reproducing amplification processes and variations in colloid sizes and shapes, but also the orientations of the adsorbed molecules on the colloid surfaces and whether they change orientation due to $\mathrm{pH}$ or other factors which may be changing. The same strange behavior is seen in C12-HSL in ABtGcasa, as can be seen in Figure 12

To be able to use SERS to observe the change in concentration of signal molecules in bacterial cell cultures the technique has to be quantitative. This behavior is not achieved in this experiment, at least not over the many decades wanted. In conventional Raman all bands would scale linearly to our concentration, which is unfortunately not the case for SERS. Furthermore, since Ag ions and Ag nanoparticles have shown to be toxic to bacteria [43] future work should use coated Ag nanoparticles. The coatings could be polymers or antibodies and be designed to capture as well as protect the bacteria. The coating proce-

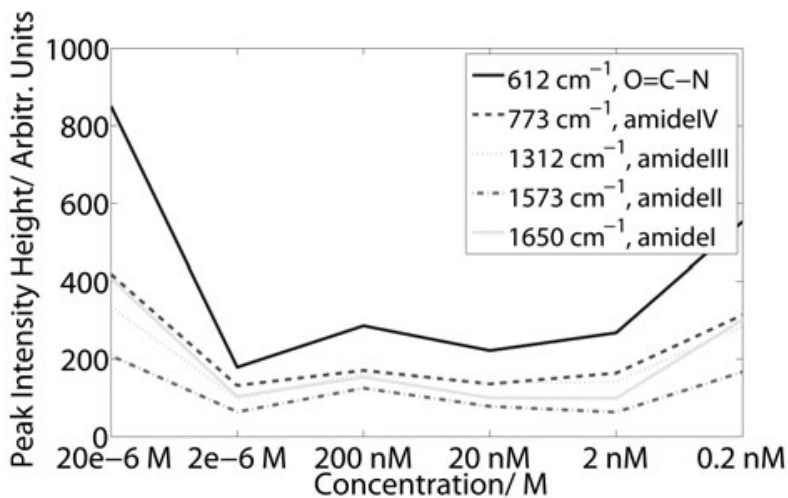

Figure 12: The peak intensity height at $612 \mathrm{~cm}^{-1}$, corresponding to the $\mathrm{O}=\mathrm{C}-\mathrm{N}$ bending vibration, is followed through the different concentrations and plotted against the concentration. Likewise are the amide bands. Here we have the plot for C12-HSL in ABtGcasa solution.

dure has been successfully used in order to measure SERS spectra of unique or rare cancer cells [44].

\section{Conclusions}

We have demonstrated that it is possible to detect the signal molecule C12-HSL down to a concentration as low as two tenths of a nM. This is obtained in water and for the first time in ABtGcasa medium. The addition of ABtGcasa medium was shown to not interfere with the vibrations of C12-HSL. The first step towards using the SERS method for in situ measurements of low AHL concentrations in biofilms is thus met. However, to be able to deduce the AHL concentration from the SERS spectrum it is necessary to have quantitative measurements. This warrants further investigation.

\section{Acknowledgments}

We thank Radiometer for the funding of the PhD-project. We are grateful to Charlotte Ijeoma Falk and Diana Chinyere Anyaogu for the support in the search for knowledge prior to the experiments.

\section{References}

[1] S.A Rice, D. McDougald, M. Givskov, and S. Kjelleberg. Detection and Inhibition of Bacterial Cell-Cell Communication. Methods Mol Biol. 2008; 
431:55-68.

[2] A.W. Decho, R.L. Frey, J.L. Ferry. Chemical Challenges to Bacterial AHL Signalling in the Environment. Chem. Rev. 2011; 111:86-99.

[3] J.F.M. Almarashi, N. Kapel, T.S. Wilkinson, and H.H. Telle. Raman Spectroscopy of Bacterial Species and Strains Cultivated under Reproducible Conditions. Spectroscopy: An International Journal 2012 27(5-6):361-365.

[4] M. Harz, P. Rösch, and J. Popp. Vibrational Spectroscopy-A Powerful Tool for the Rapid Identification of Microbial Cells at the Single-Cell Level. Cytometry A. 2009 75(2):104-13.

[5] C. Kirschner, K. Maquelin, P. Pina, N.A.N. Thi, L.P. Choo-Smith, G.D. Sockalingum, C. Sandt, D. Ami, F. Orsini, S.M. Doglia, P. Allouch, M. Mainfait, G.J. Puppels, and D. Naumann. Classification and identification of enterococci: A comparative phenotypic, genotypic, and vibrational spectroscopic study. $J$. Clin. Microbio. 2001 39(5):1763-1770.

[6] M.F. Escoriza, J.M. Vanbriesen, S. Stewart, and J. Maier. Studying bacterial metabolic states using Raman spectroscopy. Appl. Spectrosc. 2006 60(9):971976.

[7] G.D. McEwen, Y. Wu, and A. Zhou. Probing Nanostructures of Bacterial Extracellular Polymeric Substances (EPS) versus Culture Time by Raman Microspectroscopy and Atomic Force Microscopy. Biopolymers 2010 93(2):171-177.

[8] J. Bak, and J. Spanget-Larsen. Molecular and vibrational structure of the extracellular bacterial signal compound N-butyryl-homoserine lactone (C4HSL). Vibrational Spectrosc. 2009 49(2):237-241.

[9] M. Fleishmann, and P.J. Hendra, and A.J. McAuillan. Raman spectra of pyridine adsorbed at a silver electrode. Chem. Phys. Lett. 1974 26:163-166.

[10] D.L. Jeanmaire, and R.P. van Duyne. Surface raman spectroelectrochemistry: Part I. Heterocyclic, aromatic, and aliphatic amines adsorbed on the anodized silver electrode. J Electroanal Chem. 84:120.
[11] M.G. Albrecht, and J.A. Creighton. Anomalously intense Raman spectra of pyridine at a silver electrode. J Am Chem Soc. 1977 99:5215-5217.

[12] E.V. Efremov, F. Ariese and C. Gooijer. Achievements in resonance Raman spectroscopy: Review of a technique with a distinct analytical chemistry potential. Analytica Chimica Acta 2008 606(2):119134.

[13] S. Nie, and S.R. Emory. Probing Single Molecules and Single Nanoparticles by Surface-Enhanced Raman Scattering. Science 1997 275(5303):11021106.

[14] K. Kneipp, Y. Wang, H. Kneipp, L.T. Perelman, I. Itzkan, R.R. Dasari, and M.S.Feld. Single Molecule Detection Using Surface-Enhanced Raman Scattering (SERS). Phys. Rev. Lett. 1997 78(9):1667-1670.

[15] S. Efrima, and L. Zeiri. Understanding SERS of bacteria. J. Raman Spectrosc. 2008 40(3):277-288.

[16] A.Sengupta, M. Mujacic, and E.J. Davis. Detection of bacteria by surface-enhanced Raman spectroscopy. Anal Bioanal Chem. 2006 386:1379-1386

[17] R.M. Jarvis, A. Brooker, and R. Goodacre. Surfaceenhanced Raman scattering for the rapid discrimination of bacteria. Faraday Discuss. 2006 132:281292.

[18] J. Guicheteau, S. Christesen, D. Emge, and A. Tripathi. Bacterial mixture identification using Raman and surface-enhanced Raman chemical imaging. $J$. Raman Spectrosc. 2010 41:1632-1637.

[19] W.F. Pearman, M. Lawrence-Snyder, S. M. Angel, and A.W. Decho. Surface-Enhanced Raman Spectroscopy for in Situ Measurements of Signaling Molecules (Autoinducers) Relevant to Bacteria Quorum Sensing. Appl. Spectrosc. 2007 61(12):1295-1300.

[20] J. Kim, S.K. Kim, G. Grégoire, B. Manil and J.P. Schermann. Infrared Study of the Bacterial Autoinducer $N$-Hexanoyl-Homoserine Lactone (C6-HSL) in the Gas-Phase, Water, and Octanol Solutions. $J$. Phys. Chem A 2011 115:9199-9206

[21] L. Soulére, N. Guiliani, Y. Queneau, C.A Jerez and A. Doutheau. Molecular insights into quorum sensing in Acidithiobacillus ferrooxidans bacteria via molecular modelling of the transcriptional regulator AfeR and of the binding mode of long-chain acyl homoserine lactones. J. Mol. Model 2008 14:599-606 
[22] A.W. Decho, R.S. Norman and P.T. Visscher. Quorum sensing in natural environments: emerging views from microbial mats. Trends in Microbiology 2010 18(2):73-80

[23] A.W. Decho, P.T. Visscher, J. Ferry, T. Kawaguchi, L. He, K.M. Przekop, R.S. Norman and R.P. Reid. Autoinducers extracted from microbial mats reveal a surprising diversity of $\mathrm{N}$-acylhomoserine lactones (AHLs) and abundance changes that may relate to diel pH. Environmental Microbiology 2009 11(2): 409-420.

[24] D.J. Clark, and O. Maaløe. DNA replication and the division cycle in Escherichia coli. J. Mol. Biol. 1967 23(1):99-112

[25] N. Leopold, and B. Lendl. A New Method for Fast Preparation of Highly Surface-Enhanced Raman Scattering (SERS) Active Silver Colloids at Room Temperature by Reduction of Silver Nitrate with Hydroxylamine Hydrochloride. Phys. Chem. B. 2003 107:5723-5727.

[26] V. Sharma, and K. Park, and M. Srinivasarao. Colloidal dispersion of gold nanorods: Historical background, optical properties, seed-mediated synthesis, shape separation and self-assembly. Materials Science and Engineering $R 2009$ 65:1-38.

[27] S. Link, and M.A. El-Sayad. Spectral Properties and Relaxation Dynamics of Surface Plasmon Electronic Oscillations in Gold and Silver Nanodots and Nanorods. J. Phys. Chem. B 1999 103:8410-8426.

[28] T. Maiyalagan. Synthesis, characterization and electrocatalytic activity of silver nanorods towards the reduction of benzyl chloride. Applied Catalysis A: General 2008 340:191-195.

[29] G. Mie. Beiträge zur optik trüber Medien, speziell kolloidaler Metallösungen. Annalen der Physik. 1908 25:377-445.

[30] P. Chakraborty. Metal nanoclusters in glasses as nonlinear photonic materials. J. Mat. Sci 1998 33:22352249.

[31] S. Kapoor. Preparation, Characterization, and Surface Modification of Silver Particles. Langmuir 1998 14:1021-1025.

[32] A. Henglein. Reactions of Organic Free Radicals at Cilloidal Silver in Aqueous Solution. Electron Pool Effect and Water Decomposition. The Journal of Physical Chemistry 1979 83(17):2209-2216.
[33] S. Abdali, C. Johannessen, J. Nygaard and T. Norbygaard. Resonance surface enhanced Raman optical activity of myoglobin as a result of optimized resonance surface enhanced Raman scattering conditions. J. Phys. Condens. Matter. 2007 19: $285205-$ 213.

[34] M. J. Frisch, G. W. Trucks, H. B. Schlegel, G. E. Scuseria, M. A. Robb, J. R. Cheeseman, G. Scalmani, V. Barone, B. Mennucci, G. A. Petersson, H. Nakatsuji, M. Caricato, X. Li, H. P. Hratchian, A. F. Izmaylov, J. Bloino, G. Zheng, J. L. Sonnenberg, M. Hada, M. Ehara, K. Toyota, R. Fukuda, J. Hasegawa, M. Ishida, T. Nakajima, Y. Honda, O. Kitao, H. Nakai, T. Vreven, Montgomery Jr. J. A., J. E. Peralta, F. Ogliaro, M. Bearpark, J. J. Heyd, E. Brothers, K. N. Kudin, V. N. Staroverov, R. Kobayashi, J. Normand, K. Raghavachari, A. Rendell, J. C. Burant, S. S. Iyengar, J. Tomasi, M. Cossi, N. Rega, J. M. Millam, M. Klene, J. E. Knox, J. B. Cross, V. Bakken, C. Adamo, J. Jaramillo, R. Gomperts, R. E. Stratmann, O. Yazyev, A. J. Austin, R. Cammi, C. Pomelli, J. W. Ochterski, R. L. Martin, K. Morokuma, V. G. Zakrzewski, G. A. Voth, P. Salvador, J. J. Dannenberg, S. Dapprich, A. D. Daniels, . Farkas, J. B. Foresman, J. V. Ortiz, J. Cioslowski and D. J. Fox. Gaussian 09 Revision A.1. Gaussian Inc. Wallingford CT 2009.

[35] Y. Tawada, T. Tsuneda and S. Yanagisawa. A longrange-corrected time-dependent density functional theory. Journal of Chemical Physics 2004 120(18): 8425-8433.

[36] G. A. Petersson,A. Bennett, T. G. Tensfeldt, M. A. AI-Laham, and W. A. Shirley. A complete basis set model chemistry. I. The total energies of closed-shell atoms and hydrides of the first-row elements. Journal of Chemical Physics 1988 89(4): 2193-2218.

[37] G. A. Petersson and M. A. Al-Laham. A complete basis set model chemistry. II. Journal of Chemical Physics 1991 94(9): 6061-6090.

[38] A.D. Becke. Density-functional thermochemistry. III. The role of exact exchange. J. Chem. Phys. 1993 98: 5648-5652.

[39] K. Kneipp. Surface-enhanced Raman scattering. Physics Today 2007 60(11): 40-46.

[40] M. Sackmann and A. Materny. Surface enhanced Raman scattering (SERS) - a quantitative analytical tool? J. Raman Spectrosc. 2006 37: 305-310. 
[41] C. El Amri, M. Baron, and M. Maurel. Adenine and RNA in mineral samples. Surface-enhanced Raman spectroscopy (SERS) for picomole detections. Spectrochimica Acta Part A 2003 59: 2645-2654.

[42] I.T. Shadii, B.Z. Chowdhry, M.J. Snowden and R. Withnall. Semi-quantitative trace analysis of nuclear fast red by surface enhanced Raman scattering. Analytica Chimica Acta 2001 450: 115-122.

[43] N.S. Wigginton, A. De Titta, F. Piccapietra, J. Dobias, V.J.Nesatyv, M.J.F. Suter and R. BernierLatmani. Binding of silver nanoparticles to bacterial proteins depends on surface modifications and inhibits enzymatic activity. Environ. Sci. Technol. 2010 44: 2163-2168.

[44] R.A. Tripp, R.A. Dluhy and Y. Zhao. Novel nanostructures for SERS biosensing. nanotoday 2008 3: 31-37.

[45] J.P. Merrick, D. Moran and L. Radom. An Evaluation of Harmonic Vibrational Frequency Scale Factors. J. Phys. Chem. A 2007 111: 11683-11700.

[46] G. Socrates 2001. Infrared and Raman Characteristic Group Frequencies: Tables and Charts. John Wiley \& Sons Ltd, England. 2001.

[47] D. Jakubczyk, C. Barth, A. Kubas, F. Anastassacos, P. Koelsch, K. Fink, U. Schepers, G. BrennerWeiss and S. Bräse. Deuterium-labelled $N$-acylL-homoserine lactones (AHLs)-inter-kingdom signalling molecules-synthesis,structural studies, and interactions with model lipid membranes. Anal Bioanal Chem 2012 403: 473-482. 